\title{
Fish sperm made to order
}

Mice are the envy of the genetics world thanks to the ease with which these animals can be genetically altered by tinkering with cultured embryonic stem (ES) cells. When it comes to making straight transgenics, however, the mouse no longer stands apart: for this and most other species, DNA needs to be injected into embryos or germ cells, which leads to a low but nevertheless unwelcome degree of mosaicism. Kayoko Kurita and colleagues have discovered how to do away with such inefficiencies: they have successfully created transgenic sperm by genetically modifying zebrafish sperm precursors that are grown in vitro.

Hundreds of developmental zebrafish mutants have been created, and the genome sequencing project has highlighted many more genes that could be modified or inactivated. The available options for fish transgenesis, however, are

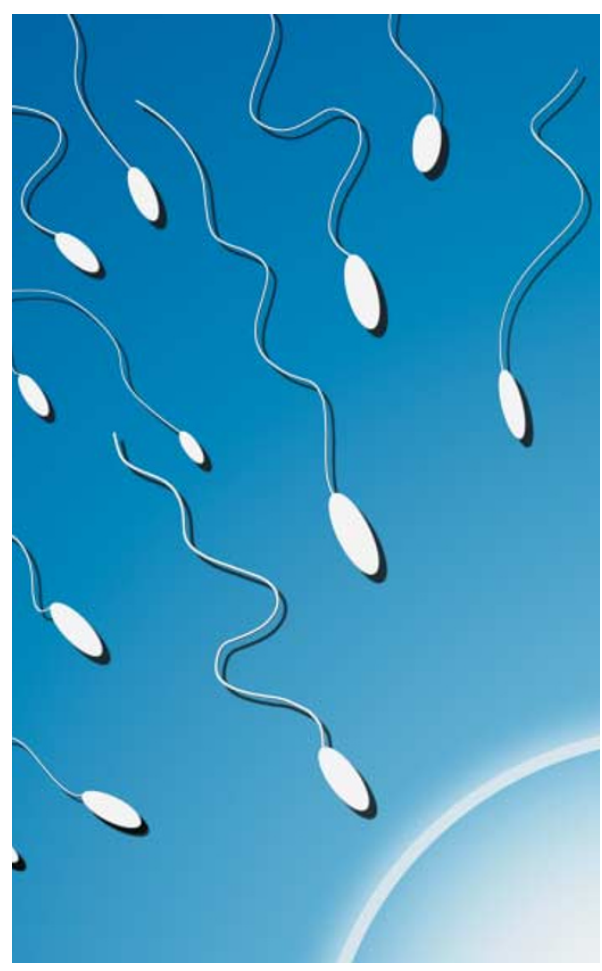

currently inadequate: for example, when DNA is injected into oocytes, embryos or the male pronucleus of a zygote, the resulting transgenic organism is usually mosaic for the transgene, meaning that germline transmission cannot be ensured until the following generation. The most straightforward way of avoiding this inconvenience would be to genetically modify sperm before fertilization, so that all cells of the $F_{1}$ progeny contain the transgene; however, this approach has not been met with much success, in vivo or in vitro, as mature sperm are refractory to transgene insertion.

To avoid the problem, Kurita and co-workers set out to introduce the transgene into sperm precursors. Primary cultures of zebrafish male germ cells were infected with retroviral vectors derived from the Moloney murine leukaemia virus. The in vitro matured sperm were then used to produce transgenic offspring. Of the 89 successfully fertilized eggs that developed into adult fish (out of 104), 5 carried the transgene. Importantly, the transgene was transmitted to offspring in a Mendelian fashion, thereby proving that the parent fish was not mosaic.

Five transgenic fish out of 89 might not seem like a vastly high rate of insertion. However, this is comparable to the rate of success of current transgenic approaches, which lead to mosaic progeny. The authors speculate that their protocol - with the appropriate tweaks - could be applied to rapidly alter the sperm genomes of other animals, including humans.

Tanita Casci

(2) References and links ORIGINAL RESEARCH PAPER Kurita, K. et al. ORIGINAL RESEARCH PAPER Kurita, K.
Transgenic zebrafish produced by retroviral infection of in vitro-cultured sperm. Proc. Natl Acad. Sci. USA 101, 1263-1267 (2004)

\section{ETHICS WATCH}

\section{The threatened trade in human ova}

It is well known that there is a shortage of human ova for in vitro fertilization (IVF) purposes, but little attention has been paid to the way in which the demand for ova in stem-cell technologies is likely to exacerbate

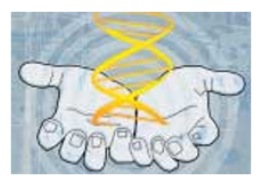
that shortfall and create a trade in human eggs. Because the 'Dolly' technology relies on enucleated ova in large quantities, allowing for considerable wastage, there is a serious threat that commercial and research demands for human eggs will grow exponentially from the combination of these two pressures. In the absence of legal regulation in the United Kingdom, and in the context of a globalized trade in human organs, we face a 'Wild West' situation in genetic and biotechnological research that involves human ova.

A recent example shows how ineffective the Human Fertilisation and Embryology Act 1990 is likely to be in regulating or stopping this trade. In a research trial, Leeds General Hospital has admitted to paying women $£ 1,500$ to undergo an IVF cycle to harvest their eggs. Commentators in the UK might have thought we would be protected from the excesses that are prevalent in the United States, where there are documented cases of extraction for profit of up to 70 ova from a single cycle in one woman (who nearly died in the process $)^{1}$. It turns out, however, that if eggs are never fertilized, the Human Fertilisation and Embryology Authority is powerless to intervene. In the Leeds case, the eggs were used by a pharmaceutical company for trials of improved techniques for in vitro maturation, during which eggs are ripened in the laboratory instead of in the ovaries. They were never fertilized. This would also be true of eggs used in stem-cell technologies.

So we face a future situation in which women in the United Kingdom are offered whatever the Local Research Ethics Committee (LREC) will condone and the 'market' will bear. That market is also likely to become globalized: already there are indications that eastern European women's ova are being extracted and sold illicitly by health-care professionals — a recent Croatian case involving two gynaecologists being a recent example. Elsewhere in eastern Europe - particularly in Russia, Bulgaria, Romania, Georgia and the Ukraine - a well-organized network that trades more generally in human organs has already been documented ${ }^{2}$. In the Leeds case, the LREC rejected an original offer by the drug company of $£ 4,000$ per woman, on the grounds that this would constitute a financial inducement rather than recompense for subjects "time and hardship". By the drug company's standards, the United Kingdom would represent a cheap market even at that price, as the going rate in the United States is US $\$ 30,000-\$ 40,000$ for one cycle's eggs. The eggs of women from eastern Europe or developing countries would presumably be even cheaper ${ }^{3}$. Unless legislation action is taken quickly to close the loophole in the Human Fertilisation and Embryology Act — that leaves unregulated the trade in eggs that are not intended to be fertilized - we face the risk of a 'free-for-all' that will imperil both women's health and the future of stem-cell research.

Donna Dickenson, Global Ethics Centre, University of Birmingham, UK e-mail:d.l.dickenson@bham.ac.uk

References ${ }^{1}$ Jacobs, A., Dwyer, J. \& Lee, P. Seventy ova. Hastings Center Report 31, 12-14 (2001) | ' Mangold, R. G. Trafficking in organs from Europe [online], (cited 15 Jan 04), $<w w w . u k t r a n s p l a n t . o r g /$ newsroom/bulletin/current_bulletin/european_legislation.htm> (2003) | ${ }^{3}$ Dickenson, D. Commodification of human tissue: implications for feminist and development ethics. Developing World Bioethics 2, 55-63 (2002) 